\title{
Multifunctionality of chiton biomineralized armor with an integrated visual system
}

\section{Citation}

Li, L., M. J. Connors, M. Kolle, G. T. England, D. I. Speiser, X. Xiao, J. Aizenberg, and C. Ortiz. 2015. Multifunctionality of Chiton Biomineralized Armor with an Integrated Visual System. Science 350, no. 6263: 952-956. doi:10.1126/science.aad1246.

\section{Published Version}

doi:10.1126/science.aad1246

\section{Permanent link}

http://nrs.harvard.edu/urn-3:HUL.InstRepos:27663225

\section{Terms of Use}

This article was downloaded from Harvard University's DASH repository, and is made available under the terms and conditions applicable to Other Posted Material, as set forth at http:// nrs.harvard.edu/urn-3:HUL.InstRepos:dash.current.terms-of-use\#LAA

\section{Share Your Story}

The Harvard community has made this article openly available.

Please share how this access benefits you. Submit a story.

\section{Accessibility}




\title{
Title: Multifunctionality of chiton biomineralized armor with an integrated visual system
}

\author{
Authors: Ling Li ${ }^{1,2 \#}$, Matthew J. Connors ${ }^{1 \#}$, Mathias Kolle ${ }^{3}$, Grant T. England ${ }^{2}$, Daniel I. Speiser ${ }^{4}$, Xianghui Xiao $^{5}$, \\ Joanna Aizenberg ${ }^{2,6,7}$, Christine Ortiz ${ }^{1, *}$
}

\section{Affiliations:}

${ }^{1}$ Department of Materials Science and Engineering, Massachusetts Institute of Technology, Cambridge, MA 021394307, USA.

${ }^{2}$ John A. Paulson School of Engineering and Applied Sciences, Harvard University, Cambridge, MA 02138, USA.

${ }^{3}$ Department of Mechanical Engineering, Massachusetts Institute of Technology, Cambridge, MA 02139, USA.

${ }^{4}$ Department of Biological Sciences, University of South Carolina, Columbia, SC 29208, USA.

${ }^{5}$ Experimental Facilities Division, Advanced Photon Source, Argonne National Laboratory, Argonne, IL 60439, USA.

${ }^{6}$ Wyss Institute for Biologically Inspired Engineering, Harvard University, Cambridge, MA 02138, USA.

${ }^{7}$ Kavli Institute for Bionano Science and Technology, Harvard University, Cambridge, MA 02138, USA.

\#These authors contributed equally to this work.

*Correspondence to: cortiz@mit.edu

\begin{abstract}
:
Nature provides a multitude of examples of multifunctional structural materials in which trade-offs are imposed by conflicting functional requirements. One such example is the biomineralized armor of the chiton Acanthopleura granulata, which incorporates an integrated sensory system that includes hundreds of eyes with aragonite-based lenses. We use optical experiments to demonstrate that these microscopic lenses are able to form images. Light scattering by the polycrystalline lenses is minimized by the use of relatively large, crystallographically-aligned grains. Multi-scale mechanical testing reveals that as the size, complexity, and functionality of the integrated sensory elements increases, the local mechanical performance of the armor decreases. However, A. granulata has evolved several strategies to compensate for its mechanical vulnerabilities to form a multi-purpose system with cooptimized optical and structural functions.
\end{abstract}

One Sentence Summary: The chiton Acanthopleura granulata is capable of seeing objects via mineral lenses integrated within its protective armor plates.

\section{Main Text:}

The design of structural materials with additional integrated functionality such as energy storage ( 1 ), sensing (2), and self-healing (3) is an emergent field that holds great potential for a diversity of engineering applications. Nature provides a multitude of multifunctional structural material systems, such as brittlestars (photosensation) (4), sponges (fiber-optic feature) (5, 6), limpets (photonic coloration) (7), and bivalves (optical transparency) $(8,9)$. Understanding the materials-level trade-offs imposed by the conflicting functional requirements of these systems is key to extracting design principles for innovative material solutions $(10,11)$.

We investigate the multifunctional design and performance of the biomineralized armor of the intertidal chiton Acanthopleura granulata, which contains an integrated visual system. Chitons are the only known group of extant mollusks to have living tissue integrated within the outermost layer of their shells (12). This tissue forms a complex network of channels that open dorsally as sensory organs known as aesthetes. A variety of functions have been 
proposed for the aesthetes (13), although observations of the phototactic behavior of a number of chitons $(14,15)$ suggest that photoreception may be a predominant role. In certain species, the aesthetes include hundreds of lens eyes $(16,17)$ that may be able to spatially resolve objects $(18)$. In stark contrast to the protein-based lenses of most animal eyes, the lenses of chitons, like their shells, are principally composed of aragonite (18). Unlike the few other eyes known to contain lenses made of calcium carbonate, such as those of trilobites $(19,20)$, the eyes of chitons are integrated within and dispersed across their entire dorsal shell surface instead of being localized to a particular region of the body. Although the calcitic lenses of brittlestars are also dispersed across their dorsal arm plates (4), it is unclear whether or not they enable spatial vision in a fashion similar to the lenses of chitons (18). Here we show that the chiton A. granulata is able to tailor the local geometry, crystallography, and interfaces of aragonite to achieve a multifunctional armor.

The two main sensory structures in the shell of A. granulata (Fig. 1A, fig. S1) appear on the surface as small bumps $\sim 50 \mu \mathrm{m}$ in diameter (Fig. 1B and C). The more numerous megalaesthetes, which are common to all chitons, maintain the same opacity as non-sensory regions and are capped with a pore, which appears as a black spot in scanning electron micrographs (SEM) (Fig. 1C). The eyes are distinguished by their translucent lenses, which are encircled by dark areas containing the pigment pheomelanin (21) (outer diameter $86 \pm 4 \mu \mathrm{m}, \mathrm{n}=10$, Fig. 1B). Both sensory structures are located within the valleys formed by the large non-sensory protrusions (diameter, $\sim 200 \mu \mathrm{m}$; height, $\sim 100 \mu \mathrm{m}$ ), as revealed by the 3D stereographic reconstruction of the shell surface (Fig. 1D and E). SEM imaging revealed that the surfaces of the lenses are much smoother than those covering the neighboring megalaesthetes and non-sensory protrusions (Fig. 1C, inset).

Synchrotron X-ray micro-computed tomography $(\mu-\mathrm{CT})$ was used to investigate the 3D morphology of the megalaesthetes and eyes (Fig. 1F and G, fig. S2, Movie S1 and S2). In contrast to the cylindrical chamber of the megalaesthetes (width of chamber, $\sim 40 \mu \mathrm{m}$ ), the specialized chamber underneath the lens is pear-shaped and has a depth and width of $56 \pm 7 \mu \mathrm{m}$ and $76 \pm 5 \mu \mathrm{m}$, respectively $(\mathrm{n}=7$, see fig. S3 for detailed morphometric measurements). This expansion results in an eye chamber whose volume is $\sim 5 \times$ greater than that of the megalaesthete. Numerous small sensory pores, known as micraesthetes, were observed branching from the chambers of the megalaesthetes and eyes to the shell surface (fig. S2, bottom). Highly X-ray absorbent structures, later determined to be intra-chamber calcified material (ICCM), were discovered within both chambers (fig. S4). In the eye, the ICCM forms a " $c$ "-shaped pocket that likely encircles the retina (Fig. 1G). The lens region of the eyes is 38 $\pm 2 \mu \mathrm{m}(\mathrm{n}=7)$ thick and slightly elongated in the direction of the optic canal, which we denote as the longitudinal direction (Fig. 1H). Fig. 1I illustrates the average cross-sectional shape of the lens in the longitudinal and transverse planes. The top and bottom surfaces of the lens were generally best fit with parabolic profiles in comparison to spherical ones (fig. S5).

Next, we compared the fine structure, composition, and crystallography of the lens region of the eyes to the bulk of the calcified portion of the outer shell layer. Polished cross sections of eyes viewed under cross-polarized light (Fig. 2A) showed that the lenses have a relatively uniform grayscale level compared to the surrounding bulk granular microstructure, which is known to have weak preferred grain orientations in the chiton Tonicella marmorea (22). This suggested that the lens is either a single crystal or is polycrystalline with highly aligned grains. The clear boundaries between the lens and granular microstructure in Fig. 2A indicate a delicate control of crystallography in the lens region. A thin ( $\sim 5 \mu \mathrm{m}$ thick) concavo-convex corneal layer covers the lens and is continuous with the surrounding granular microstructure. Sectioning an eye by focused ion beam (FIB) milling revealed the presence of two additional layers, $L 1$ and $L 2$, underlying the lens (Fig. 2B). Energy-dispersive X-ray spectroscopy (EDX) indicated that $L 1$ is mainly composed of organic materials, while $L 2$ contains calcium (Fig. 2C). Many struts branch dorsally from $L 2$ to the chamber walls (fig. S4). They correspond in size, shape, and location to the aforementioned ICCM observed in the chamber with $\mu$ CT (Fig. $1 \mathrm{G}$ ).

The crystallographic pole figures obtained with electron backscattered diffraction (EBSD) confirmed that the lens has a strong crystallographic texture, indicated by the regions of localized intensity, which is in stark contrast to the weak texture of the surrounding granular region (Fig. 2D). Integrating the $c$-axis from the pole figure of the lens shows that the full width at half maximum is $\sim 4^{\circ}$, which indicates that the $c$-axes of the grains are highly aligned (fig. S6). The high-resolution transmission electron microscopy (TEM) image and corresponding Fast Fourier Transformation (FFT) pattern from the lens (Fig. 2E) and the bright field TEM image and corresponding selected area electron diffraction (SAED) pattern of the granular microstructure (Fig. 2F) further highlight the small and large crystallographic mismatch between grains in the lens and granular microstructure, respectively. Since the effective refractive indices of aragonite are orientation-dependent, the uniform crystallographic orientation of the grains in the lens likely minimizes light scattering. EBSD and SAED of multiple lenses found that the polar angle $\theta$ 
between the $c$-axis and optical axis was about $45^{\circ}$, while the orientations of the $a$ - and $b$-axes were inconsistent (fig. S7). Since aragonite is a pseudo-uniaxial crystal, the non-normal orientation of the $c$-axes should generate double refraction, which is consistent with observations that the lenses are birefringent when viewed with polarized light $(17,18)$. In addition, EBSD showed that the lenses have an average grain size of roughly $10 \mu \mathrm{m}$, which is approximately an order of magnitude greater than that of the surrounding granular microstructure (fig. S8). Highresolution SEM images of polished cross sections display faint and clear grain boundaries in the lens and surrounding granular regions, respectively (fig. S9). The likely function of the large grains is to reduce the number and area of grain boundaries, which will minimize light scattering. Furthermore, high-resolution TEM images suggest that the lens may possess less intracrystalline organic material than the surrounding granular microstructure (fig. S10), which may also serve to reduce light scattering (8). TEM imaging and corresponding electron diffraction suggest that layers $L 1$ and $L 2$ are amorphous, despite a fine conformal layered structure observed in L2 (Fig. 2G, H).

The optical performance of individual eyes of $A$. granulata was investigated via both theoretical modeling and experimental measurements. First, key elements of the geometry, composition, and crystallography of the lens were combined in 2D ray-trace simulations to investigate the locations of rear focal points, $F$. For each possible external environment, air and seawater, $F$ of the ordinary and extraordinary rays were calculated in two orthogonal extremes, the transverse and longitudinal cross sections (Fig. 3A, see fig. S11 for detailed simulation results). The ranges of $F$ in air and seawater, $8-28 \mu \mathrm{m}$ and $25-51 \mu \mathrm{m}$ below $L 2$, respectively, lie within the geometrically permitted axial range of photoreceptive tissue, $\sim 4-52 \mu \mathrm{m}$, which is constrained above by ICCM and below by the bottom of the chamber. If $\theta$ were $0^{\circ}$ or $90^{\circ}$ instead of $45^{\circ}$, the maximum values of $F$ in seawater would be $35 \mu \mathrm{m}$ or $71 \mu \mathrm{m}$, which means the chamber would be unnecessarily large or small, respectively. Thus, the geometry of the chamber is highly consistent with $\theta \approx 45^{\circ}$.

The authors of a prior behavioral study of $A$. granulata hypothesized that the chiton may be required to use different polarizations of light to see in different tidal environments (18), In other words, only one of the two refractive indices of the birefringent lens could possibly focus light onto the photoreceptive region of the eye chamber in air, while the other does so in seawater. Although all of our simulated focal points fall within the eye chamber, we do not know the exact position and shape of the chiton retina, so we cannot conclude if birefringence is indeed functional. If this is not the case, it is puzzling why $\theta$ is not $0^{\circ}$, which would improve image quality by eliminating aberrations from double refraction as in trilobites (19) and brittlestars (4).

Since the small size (12) and perceived curvature (23) of the chiton lenses have cast doubt on their ability to form images, we decided to investigate experimentally their image formation capability by projecting objects through individual lenses in water (Fig. 3B, top). The middle image of Fig. 3B demonstrates that the lenses can form clear images of a predatory fish. These images are equivalent to those generated by a $20 \mathrm{~cm}$ long fish that is $30 \mathrm{~cm}$ away. However, the bottom pixelated image of Fig. 3B represents what the eye is probably capable of resolving, since image quality is constrained by the spacing of the photoreceptors, $s \sim 7 \mu \mathrm{m}$ (18). This suggests the maximum distance at which $A$. granulata can spatially resolve a $20 \mathrm{~cm}$ object is $\sim 2 \mathrm{~m}$, since at this object distance the image will be approximately the size of a single photoreceptor. This resolution allows chitons to quickly respond to approaching predators by clamping down to the substrate so that they are not easily dislodged (18).

The clear images produced by individual lenses allowed us to test the accuracy of our simulations. We determined the focal length, $f$, of individual eyes by measuring the dimensions of images produced from a known object at a number of object distances (Fig. 3C). The obtained $f=72 \pm 17 \mu \mathrm{m}$ is comparable to the maximum value of $f, 65 \mu \mathrm{m}$, determined from ray-trace simulations (Fig. 3A). This allowed us to quantify the resolution of an individual eye, $\Delta \varphi$, using $\Delta \varphi=\tan ^{-1}(s / f)(24)$, which ranges between $8-13^{\circ}$ in air and 6-9 ${ }^{\circ}$ in seawater, respectively. These results explain the outcome of previous behavioral experiments in which $A$. granulata was able to distinguish between dark targets with angular sizes of $\sim 9^{\circ}$ and equivalent, uniform decreases in illumination in both air and seawater (18).

Double refraction was observed during image formation experiments (fig. S12), but not consistently, which may be because the optical axes of the lens and microscope were not aligned parallel in each trial. Similarly, the extent of astigmatism observed was variable, presumably because we did not know the orientation of the transverse and longitudinal directions of each lens relative to the horizontal and vertical lines of our test objects. However, the maximum astigmatism observed, $\Delta F=19 \mu \mathrm{m}$, is consistent with the maximum, $\Delta F=17 \mu \mathrm{m}$, predicted by our raytrace simulations. Additional metrics of optical performance, including F-number, sensitivity, and field of view, can be found in fig. S13. 
Relative to the solid non-sensory regions of the outer shell layer, the integration of sensory structures introduces large, localized volumes of soft sensory tissue, and modifies the aragonite-based microstructure at the intrinsic material level. We hypothesized these changes might affect the mechanical robustness of the shell, which is surely critical to the survival of these animals. To test this hypothesis, we investigated the mechanical behavior of the outer shell layer with instrumented indentation at two length scales (Fig. 4). At the $\sim 5 \mu \mathrm{m}$ scale (maximum load $\sim 5 \mathrm{mN}$ ), both the lens and surrounding granular microstructure exhibit similar indentation modulus $\left(E_{O-P}, \sim 70 \mathrm{GPa}\right)$ and hardness $\left(H_{O-P}, \sim 5 \mathrm{GPa}\right)$ (Fig. 4A). However, nanoindentation with a sharp conospherical tip induces radial cracking in the lens region, but not in the granular microstructure (Fig. 4B). This behavior of more brittle fracture in the lens region might be due to its pseudo-single crystalline nature. To probe the mechanical behavior on the scale of the entire sensory structures, we used a flat punch tip to perform "crush" experiments on the eyes, megalaesthetes, and solid non-sensory regions (Fig. 4C). As illustrated by the load-depth curves in Fig. 4D, compression of eyes first induced gradual fracture of the protective corneal layer (Fig. 4D, inset), and eventually led to catastrophic failure by pushing the entire lens into the chamber, as shown in the post-test SEM image (Fig. 4E). The average load for the catastrophic fracture of a lens is slightly less than $1 \mathrm{~N}(0.84 \pm 0.11 \mathrm{~N})$. With a maximum load of $1 \mathrm{~N}$, the megalaesthetes exhibited step-wise micro-fracture up to the maximum load without catastrophic failure (Fig. 4E). Similar indentation on the solid non-sensory protrusions induced a relatively small amount of permanent deformation, demonstrating its greater mechanical integrity (Fig. 4E).

The structure/property/performance relationships of the shell plates of $A$. granulata demonstrate that trade-offs are present at the materials level within a single protective armor system. The shells of chitons have evolved to satisfy two conflicting design requirements, protection and sensation. Three design aspects are fundamental to the functional integration of the sensory structures within the armor: 1) the incorporation of soft sensory tissue (creation of a porous network), 2) modification of the local microscopic geometry of the armor material, and 3) the materiallevel modification of the armor material.

Sensory integration necessitates the incorporation of living tissue, which creates porosity. This degrades the mechanical robustness of the armor, which can be seen by comparing the mechanical performance of megalaesthetes to the solid non-sensory region. Depending on the species, megalaesthetes may serve a variety of functions including mechano-, chemo-, and/or photoreception (13-15). Increasing the integrated optical functionality from simple photoreception to spatial vision (in other words, advancing from light-sensitive megalaesthetes to eyes) requires a much larger volume of soft tissue per sensory unit, as well as the modification of the local geometry of the armor material to form a lens. Although the eyes provide distinct advantages over megalaesthetes, e.g. the ability distinguish the appearance of dark objects from uniform decreases in illumination, they further degrade the penetration resistance of the armor. This is demonstrated by the microindentation experiments, in which the megalaesthetes exhibited step-wise micro-fractures while the eyes failed catastrophically at less than $1 \mathrm{~N}$. Furthermore, at the material-level, increasing the grain size and alignment in the lens relative to the granular microstructure of non-sensory regions reduces scattering and improves the eye's ability to detect light (8). However, this causes the lens to fracture radially upon nanoindentation, which is in stark contrast to the relatively isotropic, localized damage observed in the non-sensory regions. These mechanical disadvantages may constrain the size of the eyes, which could improve in both resolution and sensitivity if larger (24).

Although functional integration decreases the local mechanical performance of the outer shell layer, $A$. granulata has developed strategies to compensate for its structural vulnerabilities. First, the mechanically weak sensory regions are strategically located in the valleys created by the protruding, robust non-sensory regions. This likely protects the delicate sensory structures from blunt impacts. These protrusions may also discourage fouling to make sure the sensory regions are not covered (25). Secondly, it's possible that chitons compensate for the mechanical weakness of the entire outer layer by having thick, hard underlying layers. This is consistent with observations of living chitons that had oyster-drill scars that penetrated the outer layer, but did not pierce the inner layers (26). Lastly, the apparent redundancy of the eyes helps to reduce the impact of partial shell damage. Eyes in older parts of the shell are often damaged by erosion, and replacements are continually grown at the margins of the shell plates (16). Moreover, chitons face diverse types of predatory attacks that can harm the shell plates (22). From a visual performance perspective, redundancy also ensures that $A$. granulata can simultaneously monitor the entire hemisphere for threats, which is important since the eyes are static structures and chitons can take several minutes to turn around. Additionally, redundancy may help improve sensitivity, signal-to-noise ratio, and the ability to distinguish false alarms from real threats (27). 


\section{References and Notes:}

1. J. P. Thomas, M. A. Qidwai, JOM-J. Min. Met. Mater. S. 57, 18-24 (2005).

2. Liu, et al., PNAS 110, 6694-6699 (2013).

3. Hager, et al., Adv. Mater. 22, 5424-5430 (2010).

4. J. Aizenberg, et al., Nature 412, 819-822 (2001).

5. V. C. Sundar, et al., Nature 424, 899-900 (2003).

6. J. Aizenberg, et al., PNAS 101, 3358-3363 (2004).

7. L. Li, et al., Nat. Commun. 6, doi: 10.1038/ncomms7322 (2015).

8. L. Li, C. Ortiz, Adv. Mater. 25, 2344-50 (2013).

9. L. Li, C. Ortiz, Nat. Mater. 13, 501-507 (2014).

10. J. W. C. Dunlop, P. Fratzl, Annu. Rev. Mater. Res. 40, 10.1-10.24 (2010).

11. P. Fratzl, J. W. C. Dunlop, R. Weinkamer, Eds., in Materials Design Inspired by Nature: Function through Inner Architecture (Royal Society of Chemistry, Cambridge, UK, 2013).

12. J. M. Serb, D. J. Eernisse, Evol. Educ. Outreach 1, 439-447 (2008).

13. S. Reindl, W. Salvenmoser, G. Haszprunar, J. Submicrosc. Cytol. Pathol. 29, 135-151 (1997).

14. P. Omelich, The Veliger 10, 77-82 (1967).

15. P. R. Boyle, Mar Behav Physiol. 1, 171-184 (1972).

16. H. N. Moseley, Q. J. Microsc. Sci. 25, 37-60 (1885).

17. M. Nowikoff. Zeitschrift für Wissenschaftliche Zoologie 88: 153-186 (1907).

18. D. I. Speiser, D. J. Eernisse, S. Johnsen, Curr. Biol. 21, 665-670 (2011).

19. K. M. Towe, Science 179, 1007-1009 (1973).

20. M. R. Lee, C. Torney, A. W. Owen, Palaeontology 50, 1031-1037 (2007).

21. D. I. Speiser, D. G. DeMartini, T. H. Oakley, J. Nat. Hist. 48, 45-48 (2014).

22. M. J. Connors, et al., J. Struct. Biol. 177, 314-328 (2012).

23. P. R. Boyle, Zeitschrift für Zellforsch. und mikroskopische Anat. 102, 313-332 (1969).

24. M. F. Land, D. E. Nilsson, Animal eyes (Oxford University Press, New York, 2012).

25. C. M. Kirschner, A. B. Brennan, Annu. Rev. Mater. Res. 42, 211-229 (2012).

26. L. Arey, W. Grozier, J. Exp. Zool. 29, 157-260 (1919).

27. D. E. Nilsson, Phil. Trans. R. Soc. Lond. B 346, 195-212 (1994).

Acknowledgments: We gratefully acknowledge support from the U.S. Army Research Office through the MIT Institute for Soldier Nanotechnologies (Contract W911NF-07-D-0004) and the National Security Science and Engineering Faculty Fellowship Program (N00244-09-1-0064). This work made use of the MRSEC Shared Experimental Facilities at MIT, supported by the National Science Foundation under award number DMR-0819762. Use of the Advanced Photon Source was supported by the U.S. Department of Energy, Office of Science, Office of Basic Energy Sciences, under Contract No. DE-AC02-06CH11357. M.K. and J.A. gratefully acknowledge support by the NSF DMREF program (DMR: 1533985). M.K. thanks the Alexander von Humboldt-Foundation for a Feodor Lynen Research Fellowship and gratefully acknowledges financial support from the MIT mechanical Engineering Department. D.I.S. gratefully acknowledges support by the NSF (DEB-1354831). We thank Dr. Alan Schwartzman, Dr. Yong Zhang, and Dr. Shiahn Chen for their technical assistance, and Elaine Belmonte and Bruno Anseeuw for providing photographs of $A$. granulata. D.S. collected and identified chiton specimens. M.J.C. and X.X. performed synchrotron experiments. M.J.C. and L.L. processed and analyzed data from synchrotron experiments. L.L. performed electron microscopy studies and mechanical tests with data analysis. M.J.C., G. E., M. K., and L.L. performed optical measurements and data analysis. M. K. wrote ray-tracing program. M.J.C and M. K. performed ray-trace simulations. All authors interpreted results. L.L. and M.J.C. prepared figures, tables, and movies and wrote draft manuscript. C.O. and J.A. supervised the project. All authors revised manuscript for submission. 
Figure 1:
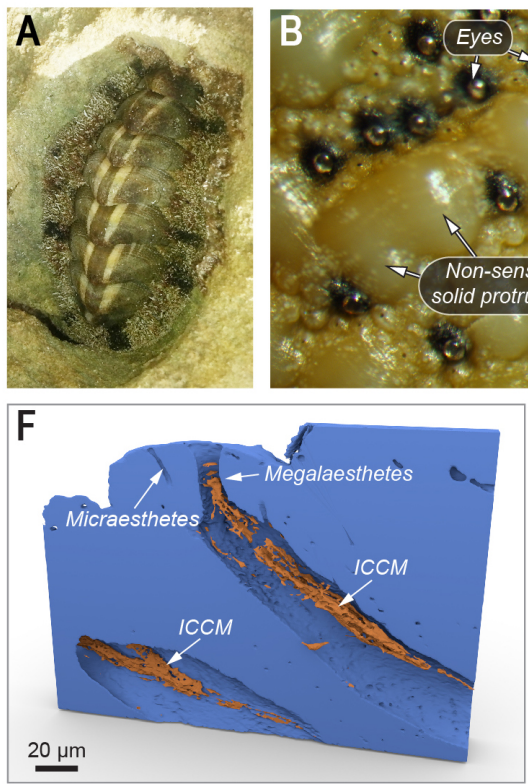

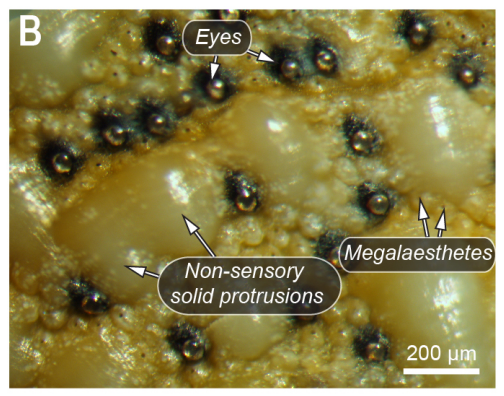

G

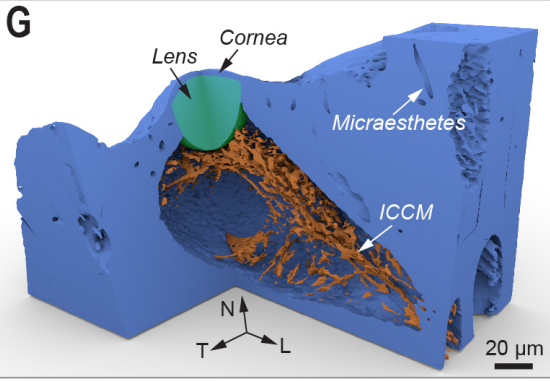

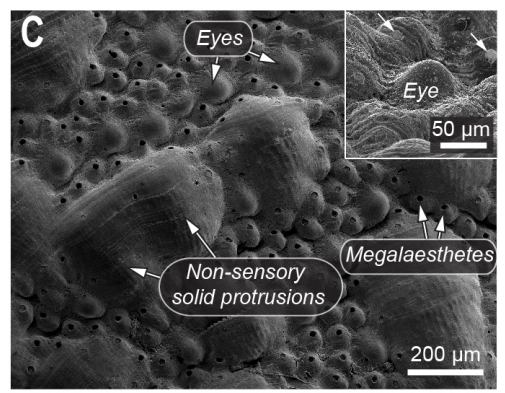
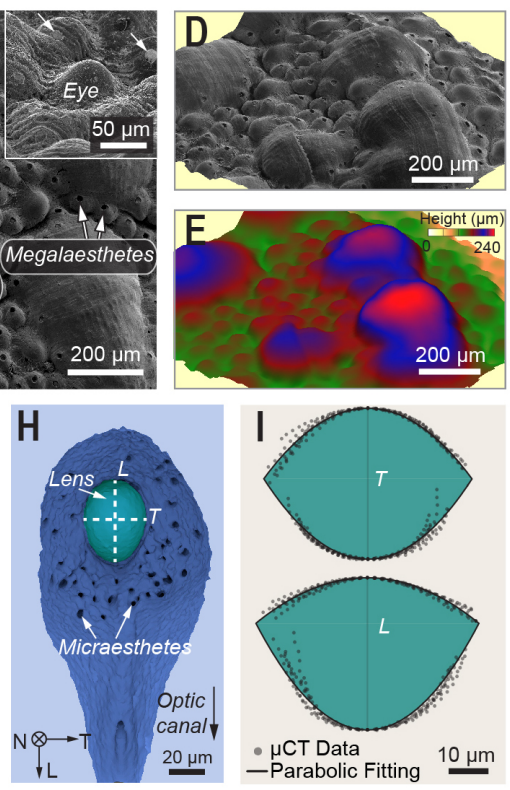

Fig. 1. Structure of the sensory elements integrated within the protective shell of the chiton Acanthopleura granulata. (A) Photograph of A. granulata. (B) Light micrograph and (C) corresponding SEM image of a region of the shell surface containing multiple eyes and megalaesthetes. The Inset of $(\mathbf{C})$ highlights the smooth and rough surfaces of the eyes and megalaesthetes (white arrows), respectively. (D and E) SEM-derived stereographic reconstruction of the shell surface: (D) surface morphology and (E) height. (F and $\mathbf{G}) 3 \mathrm{D} \mu-\mathrm{CT}$ reconstructions of a megalaesthete (F) and an eye (G), highlighting the calcified structures: outer shell layer and continuous cornea (blue), intra-chamber calcified material ("ICCM", orange), and lens (green). Numerous micraesthetes branch from the eyes and megalaesthetes. "N", "T", and "L" refer to "normal", "transverse", and "longitudinal" directions, respectively. (H) Bottom view of the lens region of (G) showing the elongated geometry along the optical canal direction (equivalent to longitudinal direction). (I) Curvature of the lenses in the " $\mathrm{T}$ " and " $\mathrm{L}$ " cross sections measured via $\mu$-CT and fittings with parabolic curves. 
Figure 2:
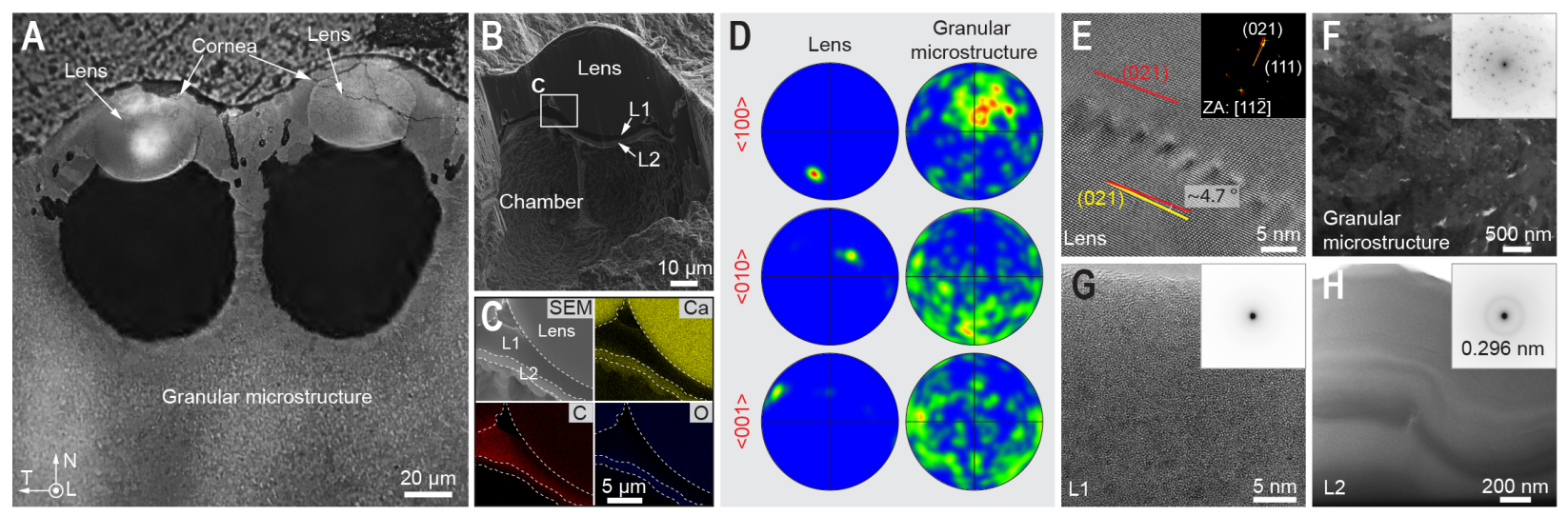

Fig.2. Structural, compositional, and crystallographic features of the lens region of the eyes. (A) Polarized light micrograph of a polished cross section containing two eyes. (B) SEM image of a FIB-cut section of an eye. (C) Energy-dispersive spectroscopy (EDS) mapping of the bottom region of the eye shown in (B). (D) Electron backscattered diffraction (EBSD) pole figures of the lens and surrounding granular microstructure in reference to the three principle orientations of aragonite. (E) High-resolution transmission electron microscopic (HRTEM) image of two adjacent aragonite grains in the lens with a small misorientation angle $\left(\sim 4.7^{\circ}\right)$. Inset, corresponding fast Fourier

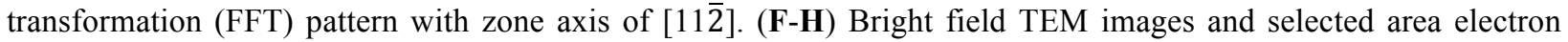
diffraction (SAED) patterns (insets) of the granular microstructure, L1, and L2, respectively. 


\section{Figure 3:}

A

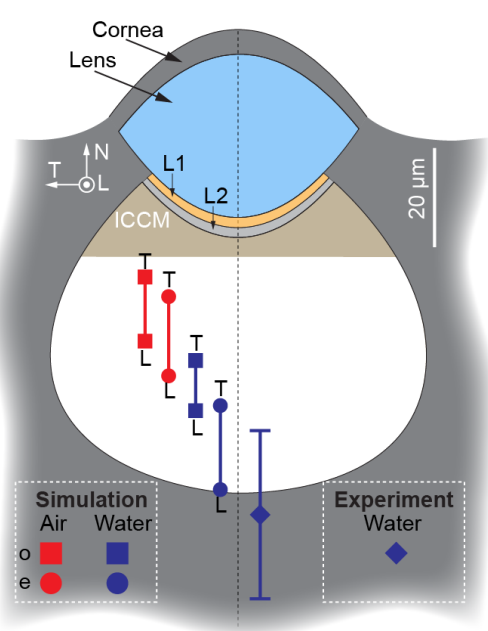

B

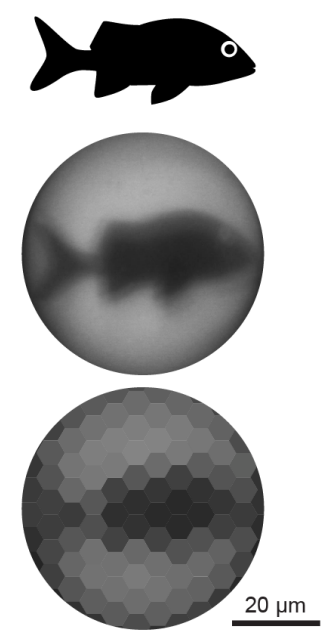

C

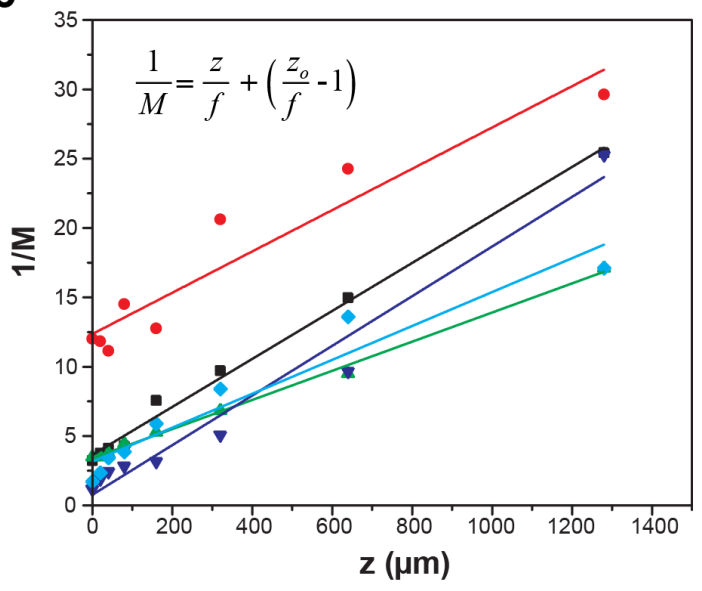

Fig.3. Focal length and image formation capacity of individual eyes. (A) Positions of the rear focal points obtained from 2D ray-trace simulations (left) and experiments (right). The red or blue color signifies an external environment of air or water, respectively. "T" and "L" indicate the cross-sectional geometry simulated. The square and circle symbols correspond to the ordinary and extraordinary rays, respectively. ICCM, intra-chamber calcified materials. The white region underneath ICCM is the location of photoreceptive cells (17). (B) Image formation ability of an individual eye. Top, the object, side profile of a predatory fish. Middle, image. Bottom, proposed physiological image resolution. Each hexagonal pixel is the size of a single photoreceptor. (C) Experimental measurements of the focal length, $f$, of 5 individual lenses derived from the slope of inverse magnification, $1 / M$, vs. object distance, $z$. 
Figure 4:
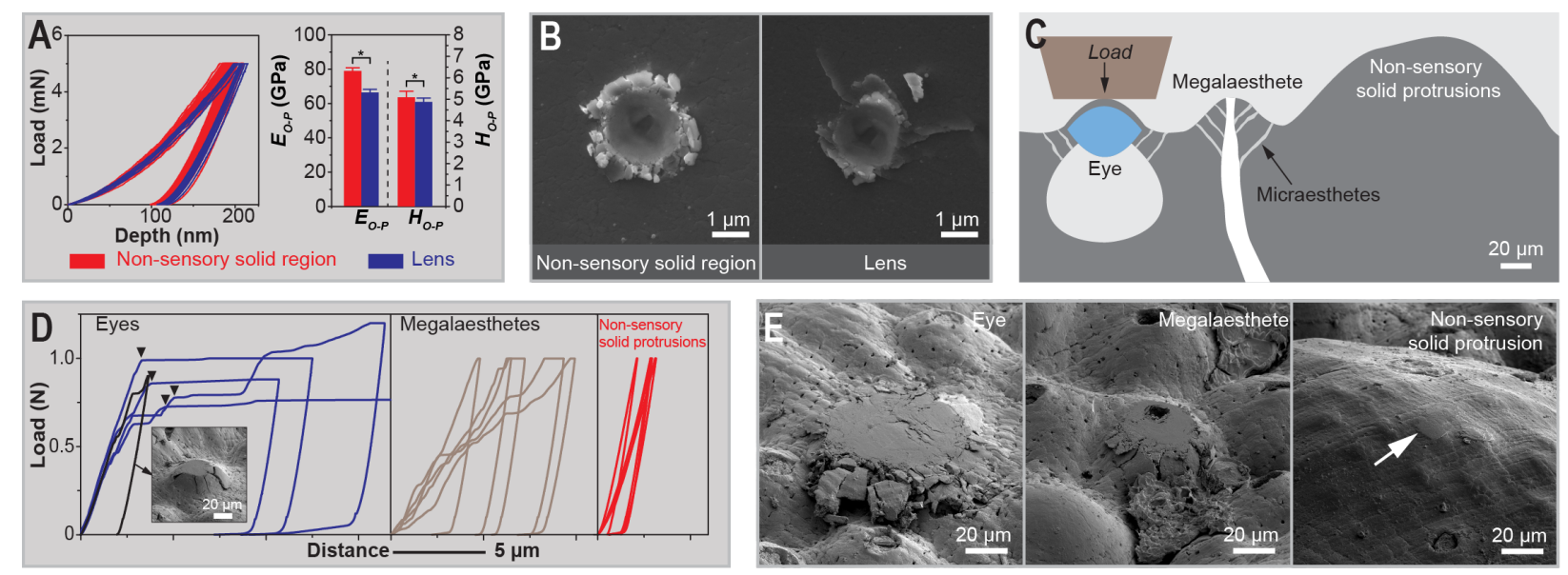

Fig.4. Trade-offs between mechanical protection and sensory integration. (A) Quantitative mechanical properties of the lens and non-sensory solid region determined through instrumented nanoindentation with a Berkovich tip. Left, the nanoindentation load-depth curves. Right, indentation modulus and hardness. "*” represents statistical significance at level of 0.05 via two sample $t$-test. The $n$ numbers for lens and non-sensory solid regions are 9 and 24, respectively. (B) SEM images of residual indents in the non-sensory region and lens, respectively, after nanoindentation with a conospherical tip (tip radius, $\sim 1 \mu \mathrm{m}$; semi-angle, $30^{\circ}$ ). (C) Schematic diagram of the microscopic compression experiments on the three areas of the outer shell layer: non-sensory regions, megalaesthetes, and eyes. (D) Microindentation force vs. depth curves for the eyes, megalaesthetes, and non-sensory regions ( $\mathrm{n}=5$ for each of the 3 structural features). The relative size and geometry of the indenter is shown in $(\mathbf{C})$. The SEM inset shows the onset of plastic deformation in the eye region, where the cornea fractures radially. (E) SEM images of residual indents in the eyes, megalaesthete, and non-sensory regions, respectively. The white arrow indicates the location of compression in the non-sensory region. 


\section{Supplementary Materials:}

Materials and Methods

Supplementary Text

Figure S1-16

Movies S1-2 